plan des trois corps auront leurs intersections communes dans un méme point.

Ce point sera le centre de gravité des trois masses, si chaque masse est augmentée proportionnellement a cube de la distancedes deux a utres.

On peut étendire ce théorème à toutes les lois possibles d'attraction, en introduisant une modification convenable daus la seconde partie de l'enoncé. En effef, soient $A_{1}, A_{2}, A_{3}$ des forces accélerafrices quelconques rapportées à l'unité de masse, qui expriment l'action nuutuelle des corps $m_{2} m_{3}$, $m_{1} m_{3}, m_{1} m_{2}$ respeclivement. Les équations du mouvement d'un des corps, par exemple de $m_{1}$, seront:

$$
\begin{aligned}
\frac{d^{2} x_{1}}{d t^{2}} & =m_{2} \frac{A_{8}}{a_{3}}\left(x_{q}-x_{1}\right)+m_{3} \frac{A_{2}}{a_{2}}\left(x_{3}-x_{1}\right), \\
\frac{d^{2} y_{1}}{d t^{2}} & =m_{2} \frac{A_{3}}{a_{3}}\left(y_{2}-y_{1}\right)+m_{3} \frac{A_{2}}{a_{2}}\left(y_{3}-y_{1}\right), \\
\frac{d^{2} z_{1}}{d t^{2}} & =m_{2} \frac{A_{3}}{a_{3}}\left(z_{2}-z_{1}\right)+m_{3} \frac{A_{2}}{a_{2}}\left(z_{3}-z_{1}\right) .
\end{aligned}
$$

En posant :

$$
\begin{gathered}
m_{1} \frac{a_{1}}{A_{1}}+m_{2} \frac{a_{2}}{A_{2}}+m_{3} \frac{a_{3}}{A_{3}}=M, \\
m_{2} \frac{a_{1}}{A_{1}} x_{1}+m_{2} \frac{a_{2}}{A_{2}} x_{2}+m_{3} \frac{a_{3}}{A_{3}} x_{3}=M X, \\
m_{1} \frac{a_{1}}{A_{1}} y_{1}+m_{2} \frac{a_{2}}{A_{2}} y_{2}+m_{3} \frac{a_{3}}{A_{3}} y_{3}=M Y, \\
m_{1} \frac{a_{1}}{A_{1}} z_{1}+m_{2} \frac{a_{2}}{A_{2}} z_{2}+m_{2} \frac{a_{3}}{A_{3}} z_{3}=M Z,
\end{gathered}
$$

ces équations deviennent:

$$
\begin{aligned}
& \frac{d^{2} x_{1}}{d t^{2}}=\frac{M}{a_{2}{ }^{3}} \cdot A_{2} \cdot A_{3}\left(X-x_{1}\right), \\
& \frac{d^{2} y_{1}}{d t^{2}}=\frac{M}{a_{2} a_{3}} A_{2} A_{3}\left(Z-y_{1}\right), \\
& \frac{d^{2} z_{1}}{d t^{2}}=\frac{M}{a_{2} a_{3}} A_{2} A_{3}\left(Z-z_{1}\right),
\end{aligned}
$$

et l'on arrive, pour les deux autres corps, à des expressions tout-à-fait symétriques.
On peut arriver à ces conclusions au moyen d'une réfléxion très-simple, qu'on peut faire. en général, pour un nombre quelconque $n$ ue corps. Supposons qu'on arrète soudainement le mouverient de ces corps, et qu' on les relie au moyen de verges infléxibles et inextensibles. On aura ainsi un système invariable, qui restera en équilibre sous l'action des attractions réciproques des différents corps. Donc si l'on considére les $n$ résultantes des attractions qui agissent sur les $n$ corps, ces résultantes seront en équilibre entre elles, et elles devront satisfaire aus conditions qui ont lieu pour un système de' $\boldsymbol{n}$ forces en équilibre.

On conclut de là, que pour le cas de trois corps, les résultantes doivent ètre contenues daus un mème plan, et passer par un mène point. Car trois forces ne sauraient étre en équilibre, si ces conditions n'ont lieu.

De meme on peut dire, d'après un théorème demontré par Möbius (Statique, $\$ \$ 98,99$ ) que pour le cas de quatre corps les quatre résultántes agissent suivant quatre génératrices d'un mêne hyperholoïde à une nappe, et que ces génératrices appartienneut au mène système. On conclut aisément de là, que le plan osculateur d'une quelconque des quatre trajectoires coupe les résultantes appliquées aux trois autres corps suivant trois points en ligne droite.

Ensuite, on pent dire, que pour cinq corps les résultants agissent suivant cinq droites telles, qu'il possibles d'en trouver deux autres qui les rencontrent toutes cinq à la fois.

Et pour six corps les droits qui contieunent les résultantes auront entre elles cette rélation de position, que M. Sylvester (Comples Rendus, vol. LII 741; appelle invoIution daus lespace.

En général, les résultantes, quelque soit leur nombre, doivent satisfaire aux six équations générales d'équilibre des systènes iurariables. Entre ces équations trois expriment que la somme des composantes des forces suivant trois directions est nulle. Les autres expriment que la somme des moments par rapport à trois axes est nulle. Des trois premières dérive le théorème de la conservation du mouvemeut du centre de gravité; des trois autres le théorème de la conservation des-aires.

\section{J. V. Schiaparelli.}

\title{
Aus einem Schreiben des Herrn Prof., Dr. Klinkerfues, Directors der Sternwarte in Göttingen, an den Herausgeber.
}

Die erste Abtheilung meines Fixstern-Catalogs ist jetzt druckfertig. Sie enthält ca. 4500 Oerter, auf 1860 reducirt; eine $\mathrm{zweite}$ nod drifte Abtheilung von nahe derselben Stärke, welche ich in nächsten Jahre zu expediren hoffen darf, wird die bis jetzt von mir angestellten Beobachtungen für diesen Zweck erledigen. Bei den Reductionen habe ich einige $\mathbf{A b}$ - 
kürzungen gefunden, in ihrer Gesammtheit bedeutend genug, die Arbeit von jetat an auf weniger als die Hälfte von der, die sie mir früher ausmachle, zu bringell. Dahei spielt folgende Transformation der bekanuten Reductionsfornelu für Herleitung des scheinbaren Ortes aus dem mittleren eine Rolle. In

$$
\begin{aligned}
& \Delta \alpha=f+g \sin (G+\alpha) t g \delta+h \sin (H+\alpha) \sec \delta, \\
& \Delta \delta=i \cos \delta+g \cos (G+\alpha)+h \cos (H+\alpha) \sin \delta
\end{aligned}
$$

setze man:

$$
\begin{aligned}
& h \sin (\boldsymbol{H}-\boldsymbol{G})=k \sin (\boldsymbol{K}-\boldsymbol{G})=\operatorname{lins}(\boldsymbol{L}+\boldsymbol{G}), \\
& g \sin (\boldsymbol{G}-\boldsymbol{H})=k \sin (\boldsymbol{K}-\boldsymbol{H})=-l \cos (\boldsymbol{L}+\boldsymbol{H}),
\end{aligned}
$$

so wird, wenn $p$ den Polar-Abstand bezeichnet:

$$
\begin{aligned}
\Delta \alpha & =f+\frac{1}{2} k \sin (\alpha+K) \operatorname{cotg} \frac{p}{2}+\frac{1}{2} l \cos (\alpha-L) \operatorname{tg} \frac{p}{2}, \\
\operatorname{secs.\Delta } \delta & =i+\frac{1}{2} k \cos (\alpha+K) \operatorname{cotg} \frac{p}{2}+\frac{1}{2} l \sin (\alpha-L) \operatorname{tg} \frac{p}{2} .
\end{aligned}
$$

Es ist $\quad k^{2}=g^{2}+h^{2}+2 g h \cos (H-G)$,

$$
l^{2}=g^{2}+h^{2}-2 g h \cos (H-G) \text {, }
$$

$k$ und $l$ sind also die Seiten zweier ebenen Dreiecke, welche beide $g$ und $h$ zu Seiten und beziehungsweise $180^{\circ}-(H-G)$ und $\boldsymbol{H}-G$ als ron den gegebenen Seiten eingeschlossene Winkel haben. Wegen der Relationen:

$$
\frac{l}{k}=\frac{\sin (K-G)}{\sin (I I-G)}, \quad \frac{g}{k}=\frac{\sin (K-H)}{\sin (G-H)}
$$

sind, wenn man yom Zeichen dieser Sinusse, d. b. von der danach vorzunehmenden Wahl des Quadranten absiebt, $\boldsymbol{K}-\boldsymbol{G}$ und $\boldsymbol{K}-\boldsymbol{H}$ ehenfalls Stücke des ersteren Dreiccks, und zwar liegen diese Winkel beziehungsweise den Seiten $\boldsymbol{k}$ und $g$ gegenüher. Dasselbe gilt von den Winkeln $90^{\circ}-(L+G)$ und $90^{\circ}-(L+H)$ in dem zweiten Dreiecke.

Die Berechnung von $k, l, K, \boldsymbol{L}$ wird wohl am kürzesten, wenn man erst

$$
\begin{aligned}
k^{2} & =(g+h)^{2} \cdot \operatorname{sen} \frac{1}{2}(H-G)^{2}+(g-h)^{2} \sin \frac{\lambda}{2}(H-G)^{2}, \\
l^{2} & =(g+h)^{2} \sin \frac{1}{2}(H-G)^{2}+(g-h)^{2} \cos \frac{2}{2}(H-G)^{2}
\end{aligned}
$$

bestimmt und dann Jurch Sulistitution $K$ und $\boldsymbol{L}$ ableitet; dahei hat man mehrere in die Augen fallende Prüfungen.

Göttingen, 1864 Juli 4.

W. Klinkerfues.

\section{Zählung der nördlichen Sterne im Bonner Sternverzeichnisse nach Grössen. Von Herrn Prolessor von Littroro.}

Durch die eben erscheioende neue Auflage der "Wunder des Hinmels" veranlasst, benutzte ich eine unir für solche Zwecke zu Gebote stehende Kraft, um während der letzten Monate die in der Ueberschrift genannte Zählung vorzunehmen. Damit

\begin{tabular}{|c|c|c|c|c|c|c|c|c|c|c|c|}
\hline \multirow{2}{*}{$\begin{array}{c}\text { Zone. } \\
\delta\end{array}$} & \multicolumn{11}{|c|}{ Grò s a e n } \\
\hline & $1-1.9$ & $2-2.9$ & $3-3.9$ & $4-4.9$ & $5-5.9$ & $6-6.9$ & $7-7.9$ & $8-8.9$ & $9-9.5$ & Neb. & Var. \\
\hline $0^{\circ}$ & . & . & 2 & 3 & 13 & 40 & 149 & 887 & 3992 & 1 & 1 \\
\hline+1 & . & . & . & 1 & 12 & 40 & 154 & 800 & 3825 & . & . \\
\hline 2 & . & . & 7 & 10 & 22 & 46 & 165 & 862 & 3640 & 1 & 1 \\
\hline 3 & . & 1 & . & 7 & 6 & 36 & 156 & 870 & $38 \dot{8}$ & & 1 \\
\hline 4 & . & . & 2 & 7 & 13 & 26 & 136 & 911 & 3997 & 1 & . \\
\hline 5 & 1 & . & 1 & 3 & 13 & 38 & 141 & 976 & 4090 & . & . \\
\hline 6 & . & 2 & 3 & 6 & 9 & 40 & 165 & 908 & 4108 & 1 & 1 \\
\hline 7 & . & . & . & 5 & 14 & 31 & 150 & 868 & 4058 & . & 3 \\
\hline 8 & 1 & . & 2 & 6 & 12 & 34 & 150 & 924 & 4041 & 1 & 4 \\
\hline 9 & . & . & 4 & 7 & 17 & 51 & 159 & $89 j$ & 4188 & . & 3 \\
\hline 10 & . & . & 4 & 3 & 13 & 47 & 178 & 882 & 3897 & - & 1 \\
\hline 11 & . & . & 2 & 3 & 22 & 51 & 184 & 923 & 3911 & 1 & . \\
\hline 12 & 1 & 1 & . & 4 & 12 & 52 & 166 & 899 & 3925 & 8 & 3 \\
\hline 13 & . & . & 2 & . & 19 & 61 & 163 & 817 & 4141 & 5 & 1 \\
\hline 14 & . & 2 & 3 & 6 & 19 & 76 & 191 & 817 & 3984 & . & 2 \\
\hline 15 & . & 1 & 4 & 3 & 14 & 78 & 206 & 808 & 3820 & 2 & 2 \\
\hline 16 & 1 & 1 & 2 & 4 & 25 & 71 & 200 & 802 & 3936 & . & 1 \\
\hline+17 & . & . & 3 & 5 & 16 & 71 & 169 & 807 & 3967 & . & . \\
\hline
\end{tabular}
gerade das kostbarste Material in Argelander's erstaunlichem Werke für die lelzten Sternclassen möglichsit in Verwendung komme, liess ich die Arbeit so ausführen, wie es die Köpfe der folgenden Columnen zeigen. Auf diese Weise wurde, nur die letzte Rubrik, die ohnehin bloss eine halbe Sterngrösse umfasst, von der Unbestimm1heit herührt, die bei Argelander für diese letzten Classen in Bezug auf Vollständigkeit der Aufnahme des Himmels herrscht; überdies war durch diese Anordnung die Zählung sehr erleichtert. 